TRANSACTIONS OF THE

AMERICAN MATHEMATICAL SOCIETY

Volume 362, Number 2, February 2010, Pages 847-858

S 0002-9947(09)04859-4

Article electronically published on September 18, 2009

\title{
REFLECTION SUBGROUPS OF COXETER GROUPS
}

\author{
ANNA FELIKSON AND PAVEL TUMARKIN
}

\begin{abstract}
We use the geometry of the Davis complex of a Coxeter group to investigate finite index reflection subgroups of Coxeter groups. The main result is the following: if $G$ is an infinite indecomposable Coxeter group and $H \subset G$ is a finite index reflection subgroup, then the rank of $H$ is not less than the rank of $G$. This generalizes earlier results of the authors (2004). We also describe the relationship between the nerves of the group and the subgroup in the case of equal rank.
\end{abstract}

\section{INTRODUCTION}

In [3, M. Davis constructed for any Coxeter system $(G, S)$ a contractible piecewise Euclidean complex, on which $G$ acts properly and cocompactly by reflections. In this paper, we use this complex to study finite index reflection subgroups of infinite indecomposable Coxeter groups from a geometrical point of view. We define convex polytopes in the complex to prove the following result:

Theorem 1.1. Let $(G, S)$ be a Coxeter system, where $G$ is infinite and indecomposable, and $S$ is finite. If $P$ is a compact polytope in $\Sigma$, then the number of facets of $P$ is not less than $|S|$.

This generalizes results of [7], where a similar result was proved for fundamental polytopes of finite index subgroups of cocompact (or finite covolume) groups generated by reflections in hyperbolic and Euclidean spaces. M. Dyer [5] proved that any reflection subgroup of a Coxeter group is also a Coxeter group. Using Theorem 1.1 and a criterion for finiteness of a Coxeter group provided by V. Deodhar [4, we obtain the main result of this paper:

Theorem 1.2. Let $(G, S)$ be a Coxeter system, where $G$ is infinite and indecomposable and $S$ is finite. Let $H \subset G$ be a finite index reflection subgroup. Then any set of reflections generating $H$ contains at least $|S|$ elements.

Further, we consider the geometry of the Davis complex itself. E. M. Andreev [1] obtained the following result for polytopes in hyperbolic and Euclidean spaces.

Received by the editors January 15, 2008.

2000 Mathematics Subject Classification. Primary 20F55, 51M20; Secondary 51F15.

The first author was supported in part by grants NSh-5666.2006.1, INTAS YSF-06-100000145916, and RFBR 07-01-00390-a.

The second author was supported in part by grants NSh-5666.2006.1, MK-6290.2006.1, INTAS YSF-06-10000014-5916, and RFBR 07-01-00390-a. 
Theorem 1.3 (Andreev [1]). Let $P$ be an acute-angled polytope in $\mathbb{E}^{n}$ or $\mathbb{H}^{n}$, and let $a$ and $b$ be two faces of $P$. If $a \cap b=\emptyset$, then the minimal planes containing, respectively, $a$ and $b$ are also disjoint.

In Section 4, we define acute-angled polytopes in the Davis complex and prove a counterpart of Andreev's theorem for non-intersecting facets of acute-angled polytopes (see Lemma 4.3).

In Section 5, we focus on the case of a Coxeter group $G$ having a finite index reflection subgroup $H$ of equal rank; i.e., this is the minimum possible rank for such an $H$. We show that the nerve of $H$ can be obtained from the nerve of $G$ by deleting some simplices (Lemma 5.1). We also give conditions on the combinatorics of $(G, S)$ which are necessary in order for $G$ to contain such an $H$ (Lemma 5.4).

We would like to thank E. B. Vinberg for useful comments and discussions and R. B. Howlett for communicating a proof of Lemma 2.1 The work was mainly done during our stay at the University of Fribourg, Switzerland. We are grateful to the University for hospitality.

\section{Preliminaries}

2.1. Coxeter systems. A Coxeter group is a group with presentation

$$
\left\langle S \mid\left(s_{i} s_{j}\right)^{m_{i j}}=1\right\rangle
$$

for all $s_{i}, s_{j} \in S$, where $m_{i i}=1$ and $m_{i j} \in\{2,3, \ldots, \infty\}$ for $i \neq j$. We further require $S$ to be finite. $S$ is called a standard generating set. A pair $(G, S)$ is called a Coxeter system.

If $S$ is fixed, $G$ is called decomposable if $S=S_{1} \cup S_{2}$, where $S_{1}$ and $S_{2}$ are non-empty subsets such that $s_{i} s_{j}=s_{j} s_{i}$ for all $s_{i} \in S_{1}, s_{j} \in S_{2}$. If $G$ is not decomposable, it is called indecomposable.

An element $s \in G$ is called a reflection if it is conjugate to some of the $s_{i} \in S$ (in particular, any $s_{i} \in S$ is a reflection). A subgroup $H \subset G$ is called a reflection subgroup of $G$ if $H$ is generated by reflections.

For any $T \subset S$ a reflection subgroup $G_{T}$ generated by all $s_{i} \in T$ is called a standard subgroup of $G$.

The rank of a Coxeter system $(G, S)$ is the number of reflections in $S$. We denote it by $|S|$.

The proof of the following lemma is suggested by R. B. Howlett.

Lemma 2.1. Let $(G, S)$ be a Coxeter system of rank $n$. Then $G$ cannot be generated by less than $n$ reflections.

Proof. Let $S=\left\{s_{1}, \ldots, s_{n}\right\}$. Consider the Tits representation of $(G, S)$ on a real vector $n$-space $V$ (see e.g. [2]). Suppose that $G$ is generated by $k<n$ reflections $r_{1}, r_{2}, \ldots, r_{k}$ along vectors $v_{1}, v_{2}, \ldots, v_{k}$, i.e.

$$
r_{i}(x)=x-2 \frac{\left(v_{i}, x\right)}{\left(v_{i}, v_{i}\right)} v_{i} .
$$

Let $L$ be the linear subspace spanned by $v_{1}, \ldots, v_{k}$. Let $g=r_{i_{1}} r_{i_{2}} \ldots r_{i_{l}}$ be any element of $G$. We prove by induction on $l$ that for any $v \in V, \quad g(v) \in v+L$. For $l=0$ the statement is evident (since $v \in v+L$ ). Suppose the statement holds for 
all elements $g=r_{i_{1}} r_{i_{2}} \ldots r_{i_{l-1}}$. Let $g=r_{i_{1}} g_{1}$, where $g_{1}=r_{i_{2}} \ldots r_{i_{l}}$. Then

$$
g(v)=r_{i_{1}} g_{1}(v)=g_{1}(v)-2 \frac{\left(v_{i_{1}}, g_{1}(v)\right)}{\left(v_{i_{1}}, v_{i_{1}}\right)} v_{i_{1}} \in v+L+\lambda v_{i_{1}} \subset v+L
$$

(where $\lambda \in \mathbb{R}$ ). Hence, $g(v) \in v+L$ for any $g \in G$.

Suppose that $g \in G$ is a reflection along some vector $v$. Then $-v=g(v) \in v+L$. Therefore, $v \in L$. Hence, by the construction of the Tits representation of $(G, S)$, $L$ should coincide with $V$, which is impossible for a space spanned by $k<n$ vectors.

2.2. Davis complex. For any Coxeter system $(G, S)$ there exists a contractible piecewise Euclidean cell complex $\Sigma(G, S)$ (called the Davis complex) on which $G$ acts discretely, properly and cocompactly. The construction was introduced by Davis 3. In 9 Moussong proved that this complex yields a natural complete piecewise Euclidean metric which is $C A T(0)$. We give a brief description of this complex following [10].

For a finite group $G$ the complex $\Sigma(G, S)$ is just one cell, which is obtained as a convex hull $C$ of $G$-orbit of a suitable point $p$ in the standard linear representation of $G$ as a group generated by reflections. The point $p$ is chosen in such a way that its stabilizer in $G$ is trivial and all the edges of $C$ are of length 1 . The faces of $C$ are naturally identified with Davis complexes of the subgroups of $G$ conjugate to standard subgroups.

If $G$ is infinite, the complex $\Sigma(G, S)$ is built up of the Davis complexes of maximal finite subgroups of $G$ gluing together along their faces corresponding to common finite subgroups. The 1-skeleton of $\Sigma(G, S)$ considered as a combinatorial graph is isomorphic to the Cayley graph of $G$ with respect to the generating set $S$.

The action of $G$ on $\Sigma(G, S)$ is generated by reflections. The walls in $\Sigma(G, S)$ are the fixed point sets of reflections in $G$. The intersection of a wall $\alpha$ with cells of $\Sigma(G, S)$ supplies $\alpha$ with a structure of a piecewise Euclidean cell complex with finitely many isometry types of cells. Walls are totally geodesic: any geodesic joining two points of $\alpha$ lies entirely in $\alpha$. Since $\Sigma$ is $C A T(0)$, any two points of $\Sigma$ may be joined by a unique geodesic.

Any wall divides $\Sigma(G, S)$ into two connected components. The complement of the union of all walls in $\Sigma(G, S)$ is a disjoint collection of connected components, and the closure of each of these components is compact; these closures are called chambers. Any chamber is a fundamental domain of $G$-action on $\Sigma(G, S)$. The set of all chambers with appropriate adjacency relation is isomorphic to the Cayley graph of $G$ with respect to $S$.

A nerve of a Coxeter system $(G, S)$ is a simplicial complex with vertex set $S$. A collection of vertices spans a simplex if and only if the corresponding reflections generate a finite group. It is easy to see that the combinatorics of the chamber of $\Sigma(G, S)$ is completely determined by the nerve: a collection of walls of a chamber has a non-empty intersection if and only if the corresponding reflections generate a finite reflection group.

For any subgroup $H \subset G$ we say that a wall $\alpha$ is a mirror of $H$ if $H$ contains the reflection with respect to $\alpha$.

In what follows, if $G$ and $S$ are fixed, we write $\Sigma$ instead of $\Sigma(G, S)$.

2.3. Convex polytopes in $\Sigma$. For any wall $\alpha$ of $\Sigma$ we denote by $\alpha^{+}$and $\alpha^{-}$the closures of the connected components of $\Sigma \backslash \alpha$; we call these components halfspaces. 
A convex polytope $P \subset \Sigma$ is an intersection of finitely many halfspaces $P=$ $\bigcap^{n} \alpha_{i}^{+}$, such that $P$ is not contained in any wall. Clearly, any convex polytope $\stackrel{i=1}{P} \subset \Sigma$ is a union of closed chambers. $P$ is compact if and only if it contains finitely many chambers. Since the walls are totally geodesic, any convex polytope is convex in the usual sense: for any two points $p_{1}, p_{2} \in P$ the geodesic segment connecting $p_{1}$ with $p_{2}$ belongs to $P$.

In what follows, by writing $P=\bigcap_{i=1}^{n} \alpha_{i}^{+}$we assume that the collection of walls $\alpha_{i}$ is minimal: for any $j=1, \ldots, n$ we have $P \neq \bigcap_{i \neq j} \alpha_{i}^{+}$. A facet of $P$ is an intersection $P \cap \alpha_{i}$ for some $i \leq n$. For any $I \subset\{1, \ldots, n\}$ a set $\bigcap_{i \in I} \alpha_{i}$ is called a face of $P$ if it is not empty.

We can easily define a dihedral angle formed by two walls: if $\alpha_{i} \cap \alpha_{j} \neq \emptyset$ there exists a maximal cell $C$ of $\Sigma$ intersecting $\alpha_{i} \cap \alpha_{j}$. We define the angle $\angle\left(\alpha_{i}, \alpha_{j}\right)$ to be equal to the corresponding Euclidean angle formed by $\alpha_{i} \cap C$ and $\alpha_{j} \cap C$. Clearly, any dihedral angle formed by two intersecting walls in $\Sigma$ is equal to $\mathrm{k \pi} / \mathrm{m}$ for some positive integers $k$ and $m$. A convex polytope $P$ is called acute-angled if each of the dihedral angles of $P$ does not exceed $\pi / 2$.

A convex polytope $P$ is called a Coxeter polytope if all its dihedral angles are integer submultiples of $\pi$. Clearly, a fundamental domain of any reflection subgroup of $G$ is a Coxeter polytope in $\Sigma$. Conversely, Theorem 4.4 of [5] implies that any Coxeter polytope in $\Sigma$ is a fundamental chamber for the subgroup of $G$ generated by reflections in its walls.

\section{Proofs of the theorems}

In what follows we write $|P|$ for the number of facets of a convex polytope $P \subset \Sigma$.

Let $a_{i}$ and $a_{j}$ be intersecting facets of a convex polytope $P \subset \Sigma$. We say that a dihedral angle of $P$ formed by $a_{i}$ and $a_{j}$ is decomposed if there exists a wall $\gamma$ containing $a_{i} \cap a_{j}$ and intersecting int $(P)$.

The following lemma is proved by V. Deodhar.

Lemma 3.1 (Deodhar 4, Proposition 4.2). Let $(G, S)$ be a Coxeter system, where $G$ is an infinite indecomposable Coxeter group and $S=\left\{s_{0}, s_{1}, \ldots, s_{k}\right\}$. Let $H=$ $\left\langle s_{i_{1}}, \ldots, s_{i_{l}}\right\rangle$ be a proper standard subgroup of $G$. Then $[G: H]=\infty$.

For the proof of Theorem 1.1 we need to consider decomposable groups also. More precisely, we use the following corollary of Lemma 3.1

Corollary 3.2. Let $(G, S)$ be a Coxeter system, where $G$ is an infinite Coxeter group and $S=\left\{s_{0}, s_{1}, \ldots, s_{k}\right\}$. Suppose that for some $l \leq k$ the group $G_{l}=\left\langle s_{0}, \ldots, s_{l}\right\rangle$ is infinite and indecomposable, and let $m$ be a positive integer not exceeding $k$. Then a subgroup $H=\left\langle s_{m}, \ldots, s_{k}\right\rangle$ has infinite index in $G$.

Proof. If $l=k$, then $G$ is indecomposable, and Lemma 3.1 applies. So, we may assume that $G$ is decomposable. Permuting elements of $S$, we may assume that there exists $p$ such that a group $G_{p}=\left\langle s_{0}, \ldots, s_{p}\right\rangle$ is indecomposable and commutes with all $s_{i}$ for $i>p$. Then the index $[G: H]$ is equal to the index $\left[G_{p}: H \cap G_{p}\right]$. Since $m \geq 1$, the subgroup $G_{p}$ is not contained in $H$, so the index $\left[G_{p}: H \cap G_{p}\right]$ is infinite due to Lemma 3.1 . 
Theorem 1.1. Let $(G, S)$ be a Coxeter system, where $G$ is infinite and indecomposable, and $S$ is finite. If $P$ is a compact polytope in $\Sigma$, then $|P| \geq|S|$.

Proof. Suppose the conclusion of the theorem does not hold. Then there exist a Coxeter system $(G, S)$ and a polytope $P$ in $\Sigma$ such that $|P|<|S|$. The proof is by induction on $|P|$, i.e. for any $k<|S|$ we assume that there is no compact polytope $P^{\prime} \subset \Sigma$ such that $\left|P^{\prime}\right|<k$ and prove that there is no compact polytope $P \subset \Sigma$ such that $|P|=k$. (Since $G$ is infinite, this trivially holds for the cases $\left|P^{\prime}\right|=0$ and 1.)

Suppose there exists a compact polytope $P \subset \Sigma$ such that $|P|=k<|S|$. Recall that $\mathcal{P}$ is the set of all compact convex polytopes in $\Sigma$. Define

$$
\mathcal{P}_{1}=\left\{P_{1} \in \mathcal{P}\left|P_{1} \subset P,\right| P_{1} \mid=k\right\} .
$$

Since $P$ is a compact polytope, $P$ is intersected by finitely many walls of $\Sigma$, so $\mathcal{P}_{1}$ is a finite set. $\mathcal{P}_{1}$ is not empty as it contains $P$. Let $P_{\min } \in \mathcal{P}_{1}$ be a polytope minimal with respect to inclusion.

Claim 1. $P_{\min }$ has no decomposed dihedral angle.

Indeed, let $a$ and $b$ be facets of $P_{\min }$, and let $\mu$ be a wall decomposing a dihedral angle formed by $a$ and $b$. Then $\mu^{+} \cap P_{\min } \in \mathcal{P}, \mu^{+} \cap P_{\min } \subset P$, and $\left|\mu^{+} \cap P_{\min }\right| \leq k$ (since $\mu^{+}$contains only one of the facets $a$ and $b$ ). By the induction assumption, the case $\left|\mu^{+} \cap P_{\min }\right|<k$ is impossible. Hence, $\left|\mu^{+} \cap P_{\min }\right|=k$, which contradicts the assumption that $P_{\min }$ is a minimal by inclusion polytope in $\mathcal{P}_{1}$.

Define the set of polytopes $\mathcal{P}_{1}^{\prime}$ in the following way:

$$
\mathcal{P}_{1}^{\prime}=\left\{P_{1}^{\prime} \in \mathcal{P}\left|P_{1}^{\prime} \subset P_{\text {min }},\right| P_{1}^{\prime} \mid=k+1,\right.
$$

and all but one facet of $P_{1}^{\prime}$ are facets of $\left.P_{\min }\right\}$.

Clearly, $\mathcal{P}_{1}^{\prime}$ is a finite set. To show that $\mathcal{P}_{1}^{\prime}$ is not empty, notice that $P_{\min }$ is not a chamber of $\Sigma$ since $\left|P_{\min }\right|=k<|S|$, while a chamber has $|S|$ facets. Therefore, there exists a wall $\mu$ decomposing $P_{\min }$ into two polytopes, namely $P_{\min } \cap \mu^{+}$and $P_{\min } \cap \mu^{-}$. It is clear that $\left|P_{\min } \cap \mu^{+}\right| \leq k+1$. The case $\left|P_{\min } \cap \mu^{+}\right|<k$ is impossible by the induction assumption, and the case $\left|P_{\min } \cap \mu^{+}\right|=k$ is impossible since $P_{\min }$ is a minimal by inclusion element of $\mathcal{P}_{1}$. Hence, $\left|P_{\min } \cap \mu^{+}\right|=k+1$, so $P_{\min } \cap \mu^{+} \in \mathcal{P}_{1}^{\prime}$.

Let $\mathcal{P}_{1 \text { min }}^{\prime} \subset \mathcal{P}_{1}^{\prime}$ be the set of polytopes of $\mathcal{P}_{1}^{\prime}$ which are minimal by inclusion, and let $P_{\min }^{\prime} \in \mathcal{P}_{1 \text { min }}^{\prime}$.

Claim 2. $P_{\min }^{\prime}$ has no decomposed dihedral angle.

Again, let $a$ and $b$ be facets of $P_{\min }^{\prime}$, and let $\mu$ be a wall decomposing the dihedral angle formed by $a$ and $b$. Then $\mu$ decomposes $P_{\min }^{\prime}$ into two polytopes, namely $P_{\min }^{\prime} \cap \mu^{+}$and $P_{\min }^{\prime} \cap \mu^{-}$. The polytope $\mu^{+} \cap P_{\min }^{\prime} \in \mathcal{P}, \mu^{+} \cap P_{\min }^{\prime} \subset P_{\min }$, and $\left|\mu^{+} \cap P_{\min }^{\prime}\right| \leq k+1$ (since $\mu^{+}$does not contain either $a$ or $b$ ). By the induction assumption, the case $\left|\mu^{+} \cap P_{\min }^{\prime}\right|<k$ is impossible. The case $\left|\mu^{+} \cap P_{\min }^{\prime}\right|=k$ is not possible either, since $P_{\min }$ is a minimal by inclusion polytope in $\mathcal{P}_{\min }^{\prime}$. Hence, $\left|\mu^{+} \cap P_{\min }^{\prime}\right|=k+1$, which contradicts the assumption that $P_{\min }^{\prime}$ is a minimal by inclusion polytope in $\mathcal{P}_{\min }^{\prime}$.

In particular, Claims 1 and 2 imply that all dihedral angles of $P_{\min }$ and $P_{\min }^{\prime}$ are equal to some dihedral angles of a chamber of $\Sigma$, so $P_{\min }$ and $P_{\min }^{\prime}$ are Coxeter polytopes. Therefore, $\left(\Gamma_{P_{\min }^{\prime}}, S_{P_{\min }^{\prime}}\right)$ is a Coxeter system for the group $\Gamma_{P_{\min }^{\prime}}$, where $S_{P_{\min }^{\prime}}$ is the complete collection of the reflections with respect to the facets of $P_{\min }^{\prime}$, 
and $\Gamma_{P_{\min }^{\prime}}$ is the group generated by all these reflections. Similarly, $\left(\Gamma_{P_{\min }}, S_{P_{\min }}\right)$ is a Coxeter system for the group $\Gamma_{P_{\min }}$, and $\Gamma_{P_{\min }}$ is a standard subgroup of $\Gamma_{P_{\min }^{\prime}}$.

The group $\Gamma_{P_{\min }}$ may be decomposable. However, any maximal indecomposable component of $\Gamma_{P_{\min }}$ is infinite. Indeed, suppose that $G_{0}=\left\langle s_{1}, \ldots, s_{l}\right\rangle$ is a maximal indecomposable standard subgroup of $\Gamma_{P_{\min }}$, and $G_{0}$ is finite. Consider the union of all polytopes $g P_{\min }, g \in G_{0}$. This union is a compact Coxeter polytope with $k-l$ facets consisting of $\left|G_{0}\right|$ copies of $P_{\min }$. This contradicts the induction assumption that we have no compact polytopes in $\Sigma$ with less than $k$ facets.

Now consider the facet $\mu$ of $P_{\min }^{\prime}$ which is not a facet of $P_{\min }$, and let us prove the following statement.

Claim 3. There exists $\widetilde{P}_{\text {min }}^{\prime} \in \mathcal{P}_{1 \text { min }}^{\prime}$ such that $\mu$ is not orthogonal to all facets of $P_{\min }$.

Suppose the contrary. Take any $P_{\min }^{\prime} \in \mathcal{P}_{1 \text { min }}^{\prime}$, and denote by $s$ the reflection in $\mu$. Then $P_{\min }^{\prime} \cup s\left(P_{\min }^{\prime}\right)=P_{\min }$; i.e., $\mu$ divides $P_{\min }$ into two congruent parts. Since $s$ commutes with all the other standard generators of $\Gamma_{P_{\min }^{\prime}}$, the group $\Gamma_{P_{\min }^{\prime}}$ is decomposable, which implies that it is a proper subgroup of $G$. In particular, $P_{\min }^{\prime}$ is not a chamber of $\Sigma$, so there is a wall $\mu_{1}$ dividing $P_{\min }^{\prime}$.

Suppose that $\mu_{1}$ is not orthogonal to all the facets of $P_{\min }$. Then consider the subset of $\mathcal{P}_{1}^{\prime}$ consisting of polytopes lying inside $P_{\min }^{\prime} \cap \mu_{1}^{+}$. This set is not empty since it contains $P_{\min }^{\prime} \cap \mu_{1}^{+}$. Take a minimal by inclusion polytope $P_{\min }^{\prime+}$. It is cut off from $P_{\min }$ by a wall $\mu_{1}^{\prime}$. By assumption, $\mu_{1}^{\prime}$ is orthogonal to all the facets of $P_{\text {min }}$. Therefore, $\mu_{1}^{\prime} \neq \mu_{1}$, and $P_{\text {min }}$ consists of two copies of $P_{\text {min }}^{\prime+}$. Now consider a subset of $\mathcal{P}_{1}^{\prime}$ consisting of polytopes lying inside $P_{\min }^{\prime} \cap \mu_{1}^{-}$, and take a minimal by inclusion polytope $P_{\min }^{\prime \prime}$. Clearly, $P_{\min }$ consists of two copies of $P_{\min }^{\prime}$ as well. However, $P_{\min }^{\prime}-$ does not intersect $P_{\text {min }}^{\prime+}$, so a copy of $P_{\min }^{\prime}-$ should contain $P_{\min }^{\prime+}$, and a copy of $P_{\min }^{\prime+}$ should contain $P_{\min }^{\prime-}$. The contradiction shows that any wall dividing $P_{\min }$ is orthogonal to all facets of $P_{\min }$.

Now consider a chamber $F$ of $\Sigma$ contained in $P_{\text {min }}$. We may assume that at least one facet of $F$ belongs to some face of $P_{\min }$. Facets of $F$ are of two types: some of them belong to facets of $P_{\min }$; the others belong to walls dividing $P_{\min }$. Both sets are non-empty. However, any facet from one of these sets is orthogonal to any facet from another one. This implies that the group $G=\Gamma_{F}$ is not indecomposable, which contradicts the assumption of the theorem.

By Claim 3, we may assume that the reflection $s$ in the facet $\mu$ of $\widetilde{P}_{\min }^{\prime}$ does not commute with all the elements of $S_{P_{\min }}$. Consider the maximal indecomposable component $\Gamma$ of $\Gamma_{\widetilde{P}_{\min }^{\prime}}$ containing $s$. Since all maximal indecomposable components of $\Gamma_{P_{\min }}$ are infinite, $\Gamma$ is also infinite. So, we are in the assumptions of Corollary 3.2. and $\left[\Gamma_{\widetilde{P}_{\min }^{\prime}}: \Gamma_{P_{\min }}\right]=\infty$. This implies that $P_{\min }$ contains infinitely many copies of $\widetilde{P}_{\text {min }}^{\prime}$; thus, $P_{\text {min }} \subset P$ contains infinitely many chambers of $\Sigma$. This contradicts the assumption that $P$ is a compact polytope in $\Sigma$.

Theorem 1.2. Let $(G, S)$ be a Coxeter system, where $G$ is infinite and indecomposable, and $S$ is finite. Let $H \subset G$ be a finite index reflection subgroup. Then any set of reflections generating $H$ contains at least $|S|$ elements.

Proof. Let $\left(H, S^{\prime}\right)$ be a Coxeter system, where $S^{\prime}$ consists of some reflections of $(G, S)$. By Lemma 2.1, any set of generating reflections of $H$ contains at least $\left|S^{\prime}\right|$ reflections. So, we are left to show that $\left|S^{\prime}\right| \geq|S|$. 
Consider a fundamental chamber $P$ of the subgroup $H$ acting on the complex $\Sigma=\Sigma(G, S)$. Since $H$ is a finite index subgroup, $P$ is a compact polytope in $\Sigma$. By Theorem 1.1 this implies that $|P| \geq|S|$. Since, $|P|=\left|S^{\prime}\right|$, we obtain the required inequality.

Remark 3.3. The conditions for $G$ to be infinite and indecomposable are essential in both Theorems 1.1 and 1.2. Clearly, any finite group contains a trivial subgroup of finite index. Concerning decomposable groups, the group

$$
\left\langle s_{1}, s_{2}, s_{3} \mid s_{1}^{2}=s_{2}^{2}=s_{3}^{2}=\left(s_{1} s_{3}\right)^{2}=\left(s_{2} s_{3}\right)^{2}=1\right\rangle
$$

contains a subgroup $\left\langle s_{1}, s_{2} \mid s_{1}^{2}=s_{2}^{2}=1\right\rangle$ of index two.

\section{ANDREEV's THEOREM}

In this section we prove a counterpart of Andreev's theorem for facets of an acute-angled polytope in the complex $\Sigma$.

Remark 4.1. In the special case where $P$ is a single chamber, then the statement is tautological even for arbitrary faces: by construction of $\Sigma$ a collection of walls (respectively, facets of a chamber) has a non-empty intersection if and only if the corresponding reflections generate a finite group. It is easy to see that the same is true if $P$ is any Coxeter polytope in $\Sigma$ : for that it is sufficient to consider $P$ as a chamber of the corresponding subgroup $\Gamma_{P}$ of $G$.

Lemma 4.2. Let $P_{1}$ and $P_{2}$ be convex polytopes in $\Sigma$ and let $c$ be a common facet of $P_{1}$ and $P_{2}$. If all the dihedral angles of $P_{1}$ and $P_{2}$ formed by $c$ with other facets of $P_{1}$ and $P_{2}$ are acute, then $P=P_{1} \cup P_{2}$ is a convex polytope.

Proof. Let $P_{1}=\gamma^{+} \cap\left(\bigcap_{i=1}^{k} \alpha_{i}^{+}\right)$and $P_{2}=\gamma^{-} \cap\left(\bigcap_{j=1}^{l} \beta_{j}^{+}\right)$, where $\gamma$ is a wall containing $c, \alpha_{i}$ is a wall containing a facet $a_{i}$ of $P_{1}$, and $\beta_{j}$ is a wall containing a facet $b_{j}$ of $P_{2}$. We will prove that $P=P_{1} \cup P_{2}=\left(\bigcap_{i=1}^{k} \alpha_{i}^{+}\right) \cap\left(\bigcap_{j=1}^{l} \beta_{j}^{+}\right)$. To prove this, it is sufficient to show that $P_{2} \subset \alpha_{i}^{+}$for all $i=1, \ldots, k$, and $P_{1} \subset \beta_{j}^{+}$for all $j=1, \ldots, l$. We prove the former of these statements; the latter may be shown in the same way. Given a facet $a_{i}=\alpha_{i} \cap P_{1}$, we consider two cases: either $\alpha_{i} \cap \gamma$ contains a face of $P$ or it does not.

At first, consider a facet $a=a_{i}$ such that $\alpha \cap \gamma$ contains a face of $P$ (where $\alpha$ is the wall containing $a$ ). Then there exists a facet $b=b_{j}$ of $P_{2}$ such that $(a \cap c) \cap P=(b \cap c) \cap P$. Denote by $\beta$ the wall containing $b$. To prove that $P_{2} \subset \alpha^{+}$, it is enough to prove that $\gamma^{-} \cap \beta^{+} \subset \alpha^{+}$. For this, it is sufficient to show that int $\left(\gamma^{-} \cap \beta^{+}\right) \cap \alpha=\emptyset$ (since $c \subset \alpha^{+}$). Since $\gamma$ divides $\Sigma$ into two connected components, $\gamma$ divides $\alpha$ into $\alpha \cap \gamma^{+}$and $\alpha \cap \gamma^{-}$. The component $\alpha \cap \gamma^{+}$ does not intersect int $\left(\gamma^{-} \cap \beta^{+}\right)$since these two sets belong to different halfspaces with respect to $\gamma$. The component $\alpha \cap \gamma^{-}$does not intersect int $\left(\gamma^{-} \cap \beta^{+}\right)$since $\alpha \cap \gamma^{-}$belongs to $\beta^{-}$(the latter statement follows immediately while considering any maximal cell of $\Sigma$ intersecting $c \cap b \cap a$ ). 
Thus, we have proved that $P \subset \alpha_{i}^{+}$for all $i \in I$, where $i \in I$ if and only if $\left(\gamma \cap \alpha_{i}\right) \cap P \neq \emptyset$. Consider $Q=\bigcap_{i \in I} \alpha_{i}^{+}$. Notice that $P_{2} \subset Q \cap \gamma^{-}$, and $\gamma \cap Q=$ $\gamma \cap P_{1}=c$.

Now, consider a facet $a_{j}$ such that $j \notin I$. Suppose that $\alpha_{j} \cap \operatorname{int}\left(P_{2}\right) \neq \emptyset$. Then $\alpha_{j} \cap\left(Q \cap \gamma^{-}\right)$is not empty either. Consider two points $p_{1} \in \operatorname{rel} \operatorname{int}\left(P_{1} \cap \alpha_{j}\right)$ and $p_{2} \in \operatorname{int}\left(P_{2}\right) \cap \alpha_{j}$. Here we require $p_{1}$ to belong to the relative interior of $P_{1} \cap \alpha_{j}$ to be sure that $p_{1}$ lies in the interior of $Q$. Clearly, $p_{2} \in \operatorname{int}(Q)$, too. Notice that there is one special case when we cannot take $p_{1} \in \operatorname{rel} \operatorname{int}\left(P_{1} \cap \alpha_{j}\right)$ : this happens if $a_{j}$ is a point. However, in this case $\alpha_{j}$ is also a point, so it cannot intersect $P_{2}$.

Consider a geodesic segment $\xi$ joining $p_{1}$ with $p_{2}$. Since $\alpha_{j}$ is a wall, and any wall is totally geodesic, $\xi \subset \alpha_{j}$. On the other hand, since $Q$ is a convex polytope and $p_{1}, p_{2} \in \operatorname{int}(Q)$, we see that $\xi \subset \operatorname{int}(Q)$. Moreover, $p_{1} \in \gamma^{+}$and $p_{2} \in \gamma^{-}$, so, there exists a point $p \in \xi$ such that $p \in \gamma$. Since $\xi \subset$ int $(Q)$, we obtain that $p \in \operatorname{int}(Q) \cap \gamma=\operatorname{int}(c)$. In particular, $\alpha_{j} \cap \operatorname{int}(c) \neq \emptyset$, which is impossible by the definition of the convex polytope $P_{1}$. Therefore, $\alpha_{j} \cap \operatorname{int}\left(P_{2}\right)=\emptyset$, and the lemma is proved.

Lemma 4.3. Let $P$ be an acute-angled polytope in $\Sigma$. Let $a$ and $b$ be facets of $P$ and $\alpha$ and $\beta$ be the walls containing $a$ and $b$ respectively. If $a \cap b=\emptyset$, then $\alpha \cap \beta=\emptyset$.

Proof. Let $r_{\alpha}$ and $r_{\beta}$ be the reflections with respect to $\alpha$ and $\beta$. Suppose that $\alpha \cap \beta \neq \emptyset$. Then, by construction of $\Sigma, r_{\alpha}$ and $r_{\beta}$ generate a finite dihedral group.

Consider a sequence of polytopes $P_{0}=P, P_{1}=r_{\alpha} P_{0}, P_{2}=r_{\alpha} r_{\beta} r_{\alpha} P_{1}=r_{\alpha} r_{\beta} P_{0}$, $P_{3}=\left(r_{\alpha} r_{\beta}\right) r_{\alpha}\left(r_{\alpha} r_{\beta}\right)^{-1} P_{2}=r_{\alpha} r_{\beta} r_{\alpha} P_{0}$, and so on, i.e. $P_{i}=r_{\alpha} r_{\beta} P_{i-2}$. By construction, $P_{i}$ and $P_{i+1}$ have a common facet, and $P_{i}$ is symmetric to $P_{i+1}$ with respect to this facet. Notice that each of the $P_{i}$ is an acute-angled polytope (as an image of an acute-angled polytope). Let $Q_{0}=P_{0}$ and $Q_{i}=Q_{i-1} \cup P_{i}$.

We claim that $Q_{i}$ is a convex polytope and any dihedral angle of $Q_{i}$ formed by $c_{i}=P_{i} \cap P_{i+1}$ and any other facet is acute. The proof is by induction on $i$. Indeed, $Q_{0}=P$ is a convex acute-angled polytope. Suppose that the statement is true for $Q_{i-1}$. Then Lemma 4.2 implies that $Q_{i}$ is a convex polytope. Any dihedral angle of $Q_{i}$ formed by $c_{i}$ and any other facet is a dihedral angle of an acute-angled polytope $P_{i}$, and hence, is acute. Therefore, $Q_{i}$ is a convex polytope for any $i$.

Notice that the wall $\gamma_{i-1}$ (containing the facet $c_{i-1}$ of $Q_{i-1}$ ) decomposes $Q_{i}$ into two convex polytopes $Q_{i} \cap \gamma_{i-1}^{+}=Q_{i-1}$ and $Q_{i} \cap \gamma_{i-1}^{-}=P_{i}$, and hence, int $\left(P_{i}\right)$ does not intersect int $\left(Q_{i-1}\right)$. On the other hand, $r_{\alpha} r_{\beta}$ has finite order, and hence, there exists $j \in \mathbb{N}$ such that $P_{j}=P_{0}$. The contradiction completes the proof of the lemma.

\section{The CASE OF EQUAL RANKS}

Throughout this section we suppose that $G$ is an infinite indecomposable Coxeter group with a finite set $S$ of standard generators, $H \subset G$ is a finite index reflection subgroup with a set $S^{\prime}$ of standard generators, and the ranks of $G$ and $H$ are equal (i.e. $\left|S^{\prime}\right|=|S|$ ). Let $\left|S^{\prime}\right|=k$.

Lemma 5.1. The nerve of a Coxeter system $\left(H, S^{\prime}\right)$ can be obtained from the nerve of $(G, S)$ by deleting some simplices. 
Proof. Let $P$ be a fundamental chamber of $H$. Define the set of polytopes $\mathcal{P}(P)$ in the following way:

$$
\mathcal{P}(P)=\left\{P^{\prime} \in \mathcal{P} \mid P^{\prime} \subset P \text {, all but one facet of } P^{\prime} \text { are facets of } P\right\} .
$$

$\mathcal{P}(P)$ is finite and non-empty: since $P$ is not a chamber of $\Sigma$, at least one wall of $\Sigma$ decomposes $P$ into two smaller polytopes, each of them belonging to $\mathcal{P}(P)$. Thus, the set $\mathcal{P}_{\min }(P)$ of polytopes minimal in $\mathcal{P}(P)$ by inclusion is not empty.

Claim. There exists $P_{1} \in \mathcal{P}_{\min }(P)$, such that $P_{1}$ is a Coxeter polytope, and $\left|P_{1}\right|=k$.

The first statement is evident: any polytope $P_{1}^{\prime} \in \mathcal{P}_{\min }(P)$ is a Coxeter polytope. Indeed, let $\mu_{1}$ be the facet of $P_{1}^{\prime}$ which is not a facet of $P$. If $P_{1}^{\prime}$ is not a Coxeter polytope, a dihedral angle formed by $\mu_{1}$ and some facet of $P$ is decomposed by some wall $\mu_{1}^{\prime}$. Then $\mu_{1}^{\prime}$ cuts out of $P$ a polytope which is contained in $P_{1}^{\prime}$, which contradicts the minimality of $P_{1}^{\prime}$.

By Theorem 1.1, any $P_{1}^{\prime} \in \mathcal{P}_{\min }(P)$ has at least $k$ facets. If it has more than $k$ facets (i.e. $k+1$ facets), then all the facets of $P$ are facets of $P_{1}^{\prime}$, so $H$ is a standard subgroup of the group $\Gamma_{P_{1}^{\prime}}$ which is generated by reflections in the facets of $P_{1}^{\prime}$.

The rest of the proof of this claim is similar to the proof of Claim 3 from the proof of Theorem 1.1 .

Suppose that any $P_{1}^{\prime} \in \mathcal{P}_{\min }(P)$ has exactly $k+1$ facets. It follows from Theorem 1.1 that any indecomposable component of $H$ is infinite. So, if for some $P_{1}^{\prime} \in \mathcal{P}_{\min }(P)$ the corresponding facet $\mu_{1}$ is not orthogonal to all the facets of $P$, then we are in the assumptions of Cor. 3.2 for the groups $\Gamma_{P} \subset \Gamma_{P_{1}^{\prime}}$. Therefore, we may assume that for any $P_{1}^{\prime} \in \mathcal{P}_{\min }(P)$ the corresponding facet $\mu_{1}$ is orthogonal to all the facets of $P$, and $P$ consists of two copies of $P_{1}^{\prime}$. In particular, $\Gamma_{P_{1}^{\prime}}$ is decomposable, so $P_{1}^{\prime}$ is not a chamber of $\Sigma$.

Now take any wall $\mu_{2}$ dividing $P_{1}^{\prime}$, and suppose that $\mu_{2}$ is not orthogonal to all the facets of $P$. Then consider two polytopes $P^{+}, P^{-} \in \mathcal{P}_{\min }(P)$ lying inside $P \cap \mu_{2}^{+}$and $P \cap \mu_{2}^{-}$respectively. $P$ consists of two copies of each of these polytopes; however, they do not intersect, so a copy of $P^{-}$should contain $P^{+}$, and a copy of $P^{+}$should contain $P^{-}$. The contradiction implies that any wall dividing $P$ is orthogonal to all the facets of $P$. Then, as in Claim 3 from the proof of Theorem 1.1, take a chamber $F$ of $\Sigma$ contained in $P$ and show that the group $G=\Gamma_{F}$ is not indecomposable. The contradiction completes the proof of the claim.

The claim above implies that we may take $P_{1} \in \mathcal{P}_{\min }(P)$ such that the corresponding facet $\mu_{1}$ does not intersect exactly one facet of $P$, say $f_{1}$. All the remaining facets of $P$ are facets of $P_{1}$. Therefore, we have a one-to-one correspondence between facets of $P$ and $P_{1}$ : facet $\mu_{1}$ corresponds to $f_{1}$, and any other facet corresponds to itself. This implies a correspondence of vertices of the nerves of $H$ and $\Gamma_{P_{1}}$. We want to prove that the nerve of $H$ can be obtained from the nerve of $\Gamma_{P_{1}}$ by deleting some simplices. For this we show that if a collection of facets of $P$ has a non-empty intersection in $P$, then the corresponding collection of facets of $P_{1}$ (i.e. substituting $\mu_{1}$ by $f_{1}$ ) has a non-empty intersection in $P_{1}$.

Suppose that a collection $J=\left\{f_{i_{1}}, f_{i_{2}}, \ldots, f_{i_{n}}\right\}$ of facets of $P$ defines a face of $P$; i.e., the intersection of all the facets contained in $J$ is not empty. If $f_{1} \notin J$, then the facets contained in $J$ have non-empty intersection in $P_{1}$ (see Remark 4.1). If $f_{1} \in J$, then consider the face $f$ of $P$ defined by the collection $J \backslash f_{1}$. By our assumption, $f$ intersects $f_{1}$. On the other hand, it was shown above that $f$ contains 
a face of $P_{1}$. Since $P_{1}$ and $f_{1}$ are contained in distinct halfspaces with respect to $\mu_{1}$, this implies that $f$ intersects $\mu_{1}$.

Therefore, the nerve of $H$ can be obtained from the nerve of $\Gamma_{P_{1}}$ by deleting some simplices. Now, substituting $P$ by $P_{1}$ in the construction above, we may choose a polytope $P_{2}$ with $k$ facets which is minimal in $\mathcal{P}\left(P_{1}\right)$. Since $P_{2} \neq P_{1}, P_{2}$ contains a smaller number of chambers of $\Sigma$ than $P_{1}$ does. Following this procedure (i.e. $P_{3}$ is minimal in $\mathcal{P}\left(P_{2}\right)$ and so on), we see that for some $m$ the polytope $P_{m}$ is a chamber of $\Sigma$, so $\Gamma_{P_{m}}=G$. The same proof as above shows that for any $i$ the nerve of $\Gamma_{P_{i}}$ can be obtained from the nerve of $\Gamma_{P_{i+1}}$ by deleting some simplices. Hence, the nerve of $H$ also can be obtained from the nerve of $G$ by deleting some simplices.

Example 5.2. As an example of the situation described in Lemma 5.1 consider a group $\Gamma=\Gamma(2,3, \infty)$ generated by reflections in the sides of a hyperbolic triangle with angles $\pi / 2, \pi / 3$ and 0 . The nerve $N$ of the group consists of 3 vertices joined by two edges, corresponding to the dihedral groups of orders 4 and 6 . This group has exactly three reflection subgroups of rank 3 . The subgroup $\Gamma_{1}=\Gamma(3,3, \infty)$ of index two is generated by reflections in the sides of a triangle with angles $\pi / 3, \pi / 3$ and 0 . Its nerve $N_{1}$ is isomorphic to $N$. The subgroup $\Gamma_{2}=\Gamma(2, \infty, \infty)$ of index three is generated by reflections in the sides of a triangle with angles $\pi / 3,0$ and 0 . Its nerve $N_{2}$ consists of 3 vertices, only two of which are joined by an edge (corresponding to a dihedral group of order 4 ). The subgroup $\Gamma_{3}=\Gamma(\infty, \infty, \infty)$ of index six is generated by reflections in the sides of an ideal triangle. Its nerve $N_{3}$ consists of 3 vertices and no edges.

Remark 5.3. Given an arbitrary Coxeter system $(G, S)$, a deleting of simplices usually does not lead to any finite index reflection subgroup. For example, it is easy to see that a group generated by reflections in the sides of an equilateral hyperbolic triangle with angles $(\pi / 5, \pi / 5, \pi / 5)$ has no finite index reflection subgroups at all. Moreover, it is not even clear when a deleting of a simplex from a nerve of $(G, S)$ corresponds to a reflection subgroup of $G$, probably of infinite index.

Finally, we provide a necessary condition for $(G, S)$ to have a finite index reflection subgroup of the same rank.

Lemma 5.4. Let $(G, S)$ be a Coxeter system, where $G$ is infinite indecomposable, and $S$ is finite. Suppose that there exists a finite index reflection subgroup $H$ of $G$ of rank $|S|$. Then there exists $s_{0} \in S$ such that at least one of the following holds:

(1) $s_{0}$ commutes with all but one element of $S$;

(2) the order of $s_{0} s$ is finite for all $s \in S$.

Proof. Let $\mathcal{P}_{k}$ be the set of polytopes in $\Sigma$ with exactly $k$ facets. Let $P_{0} \in \mathcal{P}_{k}$ be any polytope containing no elements of $\mathcal{P}_{k}$ except chambers of $\Sigma$, and $P_{0}$ itself is not a chamber of $\Sigma$. Since the rank of $H$ is equal to the rank of $G$, such a $P_{0}$ does exist (for example, one can take as $P_{0}$ a fundamental domain of $H$ in $\Sigma$ ). Clearly, there is at least one wall of $\Sigma$ which divides $P_{0}$.

Suppose that $P_{0}$ contains a decomposed dihedral angle formed by facets $f_{1}$ and $f_{2}$ of $P_{0}$. Let $\mu$ be a wall of $\Sigma$ which decomposes that angle. Then $\mu$ decomposes $P_{0}$ into two polytopes $P_{0}^{+}$, and $P_{0}^{-}$and each of them has at most $k$ facets (since one of $f_{1}$ and $f_{2}$, say $f_{2}$, is not a facet of $P_{0}^{+}$, and the other is not a facet of $P_{0}^{-}$). By Theorem 1.1, each of $P_{0}^{+}$and $P_{0}^{-}$has exactly $k$ facets. Thus, $P_{0}^{+}$and $P_{0}^{-}$are 
chambers of $\Sigma$, and $P_{0}^{+}$can be obtained from $P_{0}^{-}$by reflecting in $\mu$. Since $k-1$ of $k$ facets of $P_{0}^{+}$are facets of $P_{0}^{-}$, a facet $\mu \cap P_{0}$ of $P_{0}^{+}$is orthogonal to all but one facet of $P_{0}^{+}$, so condition (1) holds. A unique facet of $P_{0}^{+}$which is not orthogonal to $\mu \cap P_{0}$ is $f_{1}$. By assumption, $\mu$ intersects $f_{1}$, so in this case condition (2) also holds.

Now suppose that $P_{0}$ contains no decomposed dihedral angles. In particular, $P_{0}$ is a Coxeter polytope. As in the proof of Lemma 5.1 consider the set $\mathcal{P}\left(P_{0}\right)$, and take a minimal (by inclusion) element $P_{1}$. Again, $P_{1}$ is a Coxeter polytope, and $\left|P_{1}\right|=k$. By the choice of $P_{0}$, this implies that $P_{1}$ is a chamber of $\Sigma$. Let $\mu$ be the wall of $\Sigma$ which contains a facet of $P_{1}$ but contains no facets of $P_{0}$. Let $P_{2}=P_{0} \backslash P_{1}$. By Theorem 1.1, $P_{2}$ has at least $k$ facets. It is also clear that $P_{2}$ has at most $k+1$ facets.

If $P_{2}$ has $k+1$ facets, then any facet of $P_{0}$ contains a facet of $P_{2}$. Therefore, any wall containing a facet of $P_{1}$ contains a facet of $P_{2}$. Since $P_{1}$ and $P_{2}$ are contained in distinct halfspaces with respect to $\mu$, this implies that $\mu$ intersects all facets of $P_{1}$, so condition (2) holds.

If $P_{2}$ has $k$ facets, then it is also a chamber of $\Sigma$, and $P_{2}$ can be obtained from $P_{1}$ by reflecting in $\mu$. Thus, the number of facets of $P_{0}$ is the number of facets of $P_{1}$ that are orthogonal to $\mu$ plus twice the number of remaining facets of $P_{1}$ except $\mu \cap P_{0}$. Solving this linear equation, we see that $k-2$ facets of $P_{1}$ are orthogonal to $\mu$, so condition (1) holds.

Remark 5.5. Let $(G, S)$ be a Coxeter system satisfying condition (1) of Lemma 5.4 If the Coxeter relation between $s_{0}$ and $s^{\prime}$ (which is a unique generator not commuting with $s_{0}$ ) has an even exponent (or if there is no relation), then the first condition of Lemma 5.4 is also sufficient: the set $\left\{S \backslash s_{0}, s_{0} s^{\prime} s_{0}\right\}$ is a set of standard generators for a subgroup of index 2. However, in the case of an odd exponent the first condition is not sufficient: a group generated by reflections in the sides of a hyperbolic triangle with angles $(\pi / 2, \pi / 5, \pi / 5)$ has no finite index reflection subgroups of rank 3 (see, for example, 8 or $[6]$ ). The same example shows that the second condition is not sufficient either: any two standard generators of the group above generate a finite dihedral group.

\section{REFERENCES}

[1] E. M. Andreev, Intersection of plane boundaries of acute-angled polyhedra. Math. Notes 8 (1971), 761-764.

[2] N. Bourbaki, Groupes et Algèbres de Lie. Chapitres IV-VI, Hermann, Paris, 1968. MR0240238 (39:1590)

[3] M. Davis, Groups generated by reflections and aspherical manifolds not covered by Euclidean space. Ann. Math. (2) 117 (1983), 293-325. MR690848(86d:57025)

[4] V. Deodhar, On the root system of a Coxeter group. Commun. Algebra 10 (1982), 611-630. MR647210 (83j:20052a)

[5] M. Dyer, Reflection subgroups of Coxeter systems. J. Algebra 135 (1990), 57-73. MR.1076077 $(91 \mathrm{j}: 20100)$

[6] A. Felikson, Coxeter decompositions of hyperbolic polygons. Europ. J. Comb. 19 (1998), 801817. MR:1649962 (99k:52018)

[7] A. Felikson, P. Tumarkin, On finite index reflection subgroups of discrete reflection groups. Funct. Anal. Appl. 38 (2004), 313-314. MR2117513 (2005j:20046)

[8] E. Klimenko, M. Sakuma, Two-generator discrete subgroups of $\operatorname{Isom}\left(\mathbb{H}^{2}\right)$ containing orientation-reversing elements. Geom. Dedicata 72 (1998), 247-282. MR.1647707 (2000a:20111) 
[9] G. Moussong, Hyperbolic Coxeter groups. Ph.D. thesis, The Ohio State University, 1988.

[10] G. A. Noskov, E. B. Vinberg, Strong Tits alternative for subgroups of Coxeter groups. J. Lie Theory 12 (2002), 259-264. MR1885045 (2002k:20072)

Independent University of Moscow, B. Vlassievskit 11, 119002 Moscow, Russia

Current address: Department of Mathematics, University of Fribourg, Pérolles, Chemin du Musée 23, CH-1700 Fribourg, Switzerland

E-mail address: felikson@mccme.ru

Independent University of Moscow, B. Vlassievski 11, 119002 Moscow, Russia

Current address: Department of Mathematics, Michigan State University, East Lansing, Michigan 48824

E-mail address: tumarkin@math.msu.edu 